\title{
AMENABILITY, $l_{p}$-HOMOLOGIES AND TRANSLATION INVARIANT FUNCTIONALS
}

\author{
GÁBOR ELEK
}

(Received 3 June 1997; revised 28 April 1998)

Communicated by G. Robertson

\begin{abstract}
We introduce a new homology theory for infinite graphs in order to generalize some results of Willis and Woodward on translation invariant functionals. We also extend some theorems of Gerl and Gromov.
\end{abstract}

1991 Mathematics subject classification (Amer. Math. Soc.): primary 43A15; secondary 53C23.

\section{Introduction}

Let $G$ be a finite, connected, directed graph with vertex set $V(G)$ and edge set $E(G)$. A real function $f$ on $V(G)$ is called a boundary function if there exists a function $\omega$ on $E(G)$, such that $\partial \omega=f$. That is, for each $p \in V(G)$, the sum of the values of $\omega$ on the edges pointing inward $p$ minus the sum of the values of $\omega$ on the edges pointing outward $p$ is equal to $f(p)$. One of the oldest results of algebraic topology is that the codimension of boundary functions on $V$ is 1 , or in other words, the zeroth homology space of a finite, connected graph is one dimensional. Now let $G$ be an infinite, connected directed graph. The zeroth $l_{\infty}$-homology of the graph $G$ is the factor space $H_{0}^{\infty}(G)=C_{0}^{\infty}(G) / B_{0}^{\infty}(G)$, where $C_{0}^{\infty}(G)$ is the space of all bounded real functions on $V(G)$, and $B_{0}^{\infty}(G)$ is the space of functions, which are boundaries of bounded functions on the edges. According to Block and Weinberger [1], the zeroth $l_{\infty}$-homology of a graph is non-zero if and only if the graph is amenable, where the amenablility is defined using Følner sequences the same way as in the case of discrete groups. One might observe that by Poincaré-duality the above result implies the combinatorial version of Gromov's theorem on $l_{\infty}$-cohomology [4]. Namely, one

(C)1998 Australian Mathematical Society 0263-6115/98\$A2.00+0.00 
can prove that if $X$ is an $n$-dimensional, oriented combinatorial manifold, then the $n$th simplicial $l_{\infty}$-cohomology of $X$ is non-zero if and only if the dual graph of $X$ is amenable. In Sections 3 and 4 we extend the above characterization of amenability to $l_{p}$-homologies and $c_{0}$-homologies. The notion of homologies are related to the notion of translation invariant linear functionals. The $l_{p}$ and $c_{0}$ function space of discrete groups were studied by Willis $[7,8]$ and Woodward [9]. They proved that for a finitely generated group $\Gamma, l_{p}(\Gamma)(p>1)$ respectively $c_{0}(\Gamma)$ have non-zero translation invariant linear functionals if and only if $\Gamma$ is amenable. In Section 5 we show that the results of Willis and Woodward can be extended for arbitrary infinite graphs of bounded vertex degrees. For an infinite graph $G$ a translation is a bijection on $l^{\prime}(G)$, which moves the vertices by a bounded distance.

\section{The construction of a binary scheme}

In this section we show what is behind the proof of [1, Theorem 3.1]. We construct s hinan scheme for non-amenable graphs in order to facilitate the solution of certain equations. First we consider the binary tree $B$. The vertex set of $B ; V(B)$ consists if the finite $0-1$-sequences. The root vertex is the empty set $\emptyset$. It has two children, the verex indexed by 0 and the vertex indexed by 1 . In general, the vertex indexed h. $\left\{1, i_{2}, \ldots, i_{k}\right\}$ has two children: $\left\{i_{1}, i_{2}, \ldots, i_{k}, 0\right\}$ and $\left\{i_{1}, i_{2}, \ldots, i_{k}, 1\right\}$. Edges are Irju $n$ between two vertices if and only if one is the child of the other one. That is, csih vertex in $V(B)$ has degree 3 except the root, which has degree 2 . Now let $G$ te $\mathrm{d}$ connected, undirected infinite graph with bounded vertex degrees. Its vertex set 1. equipped with the path-distance metric $d_{G}: V(G) \times V(G) \rightarrow \mathbb{N}$. We call such a graph a nice graph.

Let $U \subset V(G)$, then $B(U, k)=\left\{x \in V(G): \min _{y \in U} d_{G}(x, y) \leq k\right\}$. The nice graph $G$ is amenable [1], if there exists a sequence of finite sets $\left\{U_{i}\right\}_{i>0}$, such that

(1)

$$
\lim _{n \rightarrow \infty} \frac{\left|B\left(U_{n}, 1\right)\right|}{\left|U_{n}\right|}=1
$$

where $\left|U_{n}\right|$ denotes the cardinality of $U_{n}$. If $G$ is the Cayley-graph of a finitely generated amenable group then $\left\{U_{i}\right\}_{i>0}$ can be chosen as an exhaustion of $G$ [1]. Obviously, if $G$ is non-amenable, then there exists a constant $l \in \mathbb{N}$ such that for any finite non-empty set $U \subset V(G)$ :

$$
\frac{|B(U, l)|}{|U|} \geq 2
$$

Let $G$ be a nice graph. A binary scheme on $G$ is a map $P: V(G) \times V(B) \rightarrow V(G)$ such that: 
(i) for any $x \in V(G)$, the map $P(x,$.$) is injective and P(x, \emptyset)=x$, where $\emptyset$ is the root vertex of the binary graph $B$;

(ii) there exists a constant $m \in \mathbb{N}$ such that for any $x \in V(G)$ and adjacent vertices $a, b \in V(B)$,

$$
d_{G}(P(x, a), P(x, b)) \leq m
$$

(iii) for any $y \in V(G)$, the inverse image of $y, P^{-1}(y)$, consists of at most two elements.

PROPOSITION 2.1. If $G$ is a nice, non-amenable graph, then there exists a binary scheme $P: V(G) \times V(B) \rightarrow V(G)$.

PROOF. The proof is based on the following immediate corollary of $[2$, Theorem 4.1].

LEMMA 2.2. There exist maps $\psi_{1}, \psi_{2}, \psi_{3}, \psi_{4}$ from $V(G)$ to $V(G)$, such that

(i) if $\psi_{i}(x)=\psi_{j}(y)$, then $x=y$ and $i=j$;

(ii) for some $n>0, d\left(\psi_{i}(x), x\right)<n$ uniformly on $\{1,2,3,4\} \times V(G)$.

Now we define the map $P$ by an inductive process using the $\psi_{i}$ 's of Lemma 2.2 . Let $a_{0}, a_{1}, a_{2}, \ldots$ be an enumeration of $V(G)$.

The first step is:

$$
P\left(a_{0}, \emptyset\right)=a_{0}, P\left(a_{0},\{0\}\right)=\psi_{3}\left(a_{0}\right), P\left(a_{0},\{1\}\right)=\psi_{4}\left(a_{0}\right) .
$$

The second step is as follows:

$$
\begin{gathered}
P\left(a_{0},\{0,0\}\right)=\psi_{1}\left(P\left(a_{0},\{0\}\right)\right), P\left(a_{0},\{0,1\}\right)=\psi_{2}\left(P\left(a_{0},\{0\}\right)\right) \\
P\left(P\left(a_{0},\{0\}\right), \emptyset\right)=P\left(a_{0},\{0\}\right), P\left(P\left(a_{0},\{0\}\right),\{0\}\right)=\psi_{3}\left(P\left(a_{0},\{0\}\right),\right. \\
P\left(P\left(a_{0},\{0\}\right),\{1\}\right)=\psi_{4}\left(P\left(a_{0},\{0\}\right)\right. \\
P\left(a_{0},\{1,0\}\right)=\psi_{1}\left(P\left(a_{0},\{1\}\right)\right), P\left(a_{0},\{1,1\}\right)=\psi_{2}\left(P\left(a_{0},\{1\}\right)\right) \\
P\left(P\left(a_{0},\{1\}\right), \emptyset\right)=P\left(a_{0},\{1\}\right), P\left(P\left(a_{0},\{1\}\right),\{0\}\right)=\psi_{3}\left(P\left(a_{0},\{1\}\right),\right. \\
P\left(P\left(a_{0},\{1\}\right),\{1\}\right)=\psi_{4}\left(P\left(a_{0},\{1\}\right) .\right.
\end{gathered}
$$

Still during the second step, we choose the first element $a_{i}$ in the sequence $a_{0}, a_{1}, \ldots$ which is not yet in the image of $P$ and define: $P\left(a_{i}, \emptyset\right)=a_{i}, P\left(a_{i},\{0\}\right)=\psi_{3}\left(a_{i}\right)$, $P\left(a_{i},\{1\}\right)=\psi_{4}\left(a_{i}\right)$.

For the $k$-step, let us consider the set $\left\{y_{1}, y_{2}, \ldots y_{s}\right\} \subset V(G)$ with the following properties: 
(i) For any $1 \leq i \leq s$, there exists $x_{i} \times b_{i} \in V(G) \times V(B)$, such that $P\left(x_{i}, b_{i}\right)=y_{i}$ has already been defined in the first $k-1$ steps.

(ii) On the other hand, $P\left(x_{i}, c_{i}\right)$ and $P\left(x_{i}, d_{i}\right)$ are still undefined, where $c_{i}, d_{i}$ are the children of $b_{i}$.

Note that for any $y_{i}$, such a pair $\left(x_{i}, b_{i}\right)$ is unique by our construction. Then for each $y_{i}$, we define:

$$
\begin{aligned}
& P\left(x_{i}, c_{i}\right)=\psi_{1}\left(y_{i}\right), P\left(x_{i}, d_{i}\right)=\psi_{2}\left(y_{i}\right), \\
& P\left(y_{i}, \emptyset\right)=y_{i}, P\left(y_{i},\{0\}\right)=\psi_{3}\left(y_{i}\right), P\left(y_{i},\{1\}\right)=\psi_{4}\left(y_{i}\right) .
\end{aligned}
$$

Now we choose again the first $a_{j}$ in the enumeration of $V(G)$, which is not yet defined to be in the image of $P$, and let

$$
P\left(a_{j}, \emptyset\right)=a_{j}, P\left(a_{j},\{0\}\right)=\psi_{3}\left(a_{j}\right), P\left(a_{j},\{1\}\right)=\psi_{4}\left(a_{j}\right) .
$$

Inductively we can define $P$ for all $(x, b) \in V(G) \times V(B)$.

\section{Homology theories}

The notion of uniformly bounded homology was introduced by Block and Weinberger [1]. We need an analogue of their construction. Let $G$ be a nice graph. The vertex set $V(G)$ is a metric space and one can equip $V(G)^{i+1}$, the $(i+1)$-fold Cartesian product of $V(G)$, with a metric as well:

$$
d\left(\left(x_{0}, x_{1}, \ldots, x_{i}\right),\left(y_{0}, y_{1}, \ldots, y_{i}\right)\right)=\max _{0 \leq j \leq i} d\left(x_{j}, y_{j}\right) .
$$

Denote by $\Delta_{i+1}$ the multidiagonal in $V(G)^{i+1}$. For $1<p<\infty$, let $C_{i}^{l_{p}}(G)$ be the vector space of infinite formal sums $c=\sum a_{\bar{x}} \bar{x}$, where $\bar{x} \in V(G)^{i+1}, a_{\bar{x}} \in \mathbb{R}$ satisfying the following two conditions:

(i) $\sum\left|a_{\bar{x}}\right|^{p}<\infty$;

(ii) there exists $R>0$ (depending only on $c$ ) such that $a_{\bar{x}}=0$, if $d\left(\bar{x}, \Delta_{i+1}\right)>R$. The usual boundary operator $\partial: C_{i}^{l_{p}}(G) \rightarrow C_{i_{1}}^{l_{p}}(G)$ is defined by

$$
\partial\left(x_{0}, x_{1}, \ldots, x_{i}\right)=\sum_{j=0}(-1)^{j}\left(x_{0}, x_{1}, \ldots, \hat{x_{j}}, \ldots, x_{i}\right) .
$$

Then $\partial \partial=0$, hence $\left(C_{*}^{f_{p}}(G), \partial\right)$ is a chain complex. The homology of this complex is the $l_{p}$-homology of $G, H_{i}^{l_{p}}(G)$. The $c_{0}$-homology of $G: H_{i}^{c_{0}}(G)$ can be defined analogously. We only need to replace condition (1) with the following one:

$$
a_{\bar{x}} \rightarrow 0 \text { as } d_{G}\left(x_{0}, q\right) \rightarrow \infty \text {, where } q \text { is a fixed vertex of } G .
$$


If $H$ is another nice graph and $f: V(G) \rightarrow V(H)$ is a quasi-isometry in the sense of Gromov, then we can define $f_{*}: C_{i}^{l_{F}}(G) \rightarrow C_{i}^{l_{p}}(H)$ and $f_{*}: C_{i}^{c_{0}}(G) \rightarrow C_{i}^{c_{0}}(H)$ by

$$
f_{*}\left(\sum a_{\bar{x}} \bar{x}\right)=\sum a_{\bar{x}} f(\bar{x}) .
$$

Following the argument of [1], one can see that $f_{*}$ induces an isomorphism between $H_{i}^{l_{p}}(G)$ and $H_{i}^{l_{p}}(H)$, and between $H_{i}^{c_{0}}(G)$ and $H_{i}^{c_{0}}(H)$.

A very important observation of Block and Weinberger is that the Oth uniformly finite homology of $G$ is zero if and only if $G$ is non-amenable. Analogously, we have the following theorem.

THEOREM 1. For a nice graph $G$, the following conditions are equivalent.

(i) $G$ is non-amenable;

(ii) $H_{0}^{l_{p}}(G)=0$;

(iii) $H_{0}^{c_{0}}(G)=0$.

Proof. Let $G$ be a nice non-amenable graph. Then by Proposition 2.1 we have a binary scheme $P: V(G) \times V(B) \rightarrow V(G)$. Let $\delta_{0}$ be the characteristic function of the root vertex $B$. Then we consider the following 1-chain $\Psi=\sum a_{x, y}(x, y)$ on $B$ :

$$
a_{\{,\{0\}}=-\frac{1}{2}, a_{\{,\{1\}}=-\frac{1}{2}, a_{\{0\},\{01\}}=-\frac{1}{4}, \ldots, a_{\left\{i_{1}, i_{2}, \ldots, i_{k-1}\right\},\left\{i_{1}, i_{2}, \ldots . i_{k-1}, i_{k}\right\}}=-\frac{1}{2^{k}},
$$

all other coefficients $a_{x, y}$ are zeroes. Then of course, $\partial \Psi=\delta_{0}$.

Now let $f$ be a function on $V(G)$. For any $x \in V(G)$, we define $\Phi_{x}=P(x, .)_{*}(\Psi)$. Then $\partial \Phi_{x}=\delta_{x}$, the characteristic function of $x$. Hence one can define $\Phi^{f}=$ $\sum_{x \in V(G)} f(x) \Phi_{x}$. It is easy to see that $\partial \Phi^{f}=f$. Moreover, if $f \in l_{p}(G),(p>1)$ then $\Phi^{f} \in C_{1}^{l_{n}}(G)$; and if $f \in c_{0}(G)$ then $\Phi^{f} \in C_{1}^{c_{0}}(G)$. It is important to note that $\Phi^{f} \notin C_{1}^{l_{1}}(G)$.

The proof of the 'only if' part is a little bit more complicated than in the case of uniformly finite chains [1], but the idea is similar. Suppose that $G$ is a nice amenable graph. There exists a sequence of finite disjoint sets $U_{1}, U_{2}, \ldots$ such that

$$
\frac{\left|B\left(U_{n}, 1\right)\right|}{\left|U_{n}\right|} \leq 1+2^{-n}
$$

First we consider the $l_{p}$-case $(p>1)$. Let $L_{n}=n^{-2 / p}\left|U_{n}\right|^{-1 / p}$ and let $\delta_{n}$ be the characteristic function of $U_{n}$. Let $f=\sum_{n=1}^{\infty} L_{n} \delta_{n}$. Then

$$
\sum_{x \in V(\mathcal{G})}|f(x)|^{p}=\sum_{n=1}^{\infty}\left|U_{n}\right| L_{n}^{p}=\sum_{n=1}^{\infty} \frac{1}{n^{2}}<\infty .
$$


That is, $f \in l_{p}(G)=C_{0}^{l_{n}}(G)$. Suppose that $f=\partial \Phi$, where $\Phi=\sum a_{x . y}(x, y) \in$ $C_{1}^{l_{p}}(G)$. Then

$$
L_{n}\left|U_{n}\right|=\sum_{x \in U_{n}} \partial \Phi(x)=\sum_{x \in U_{n}} \sum_{y \in V(G)}\left(-a_{x, y}+a_{y, x}\right)=\sum_{x \in U_{n}} \sum_{y \notin U_{n}}\left(-a_{x, y}+a_{y, x}\right) .
$$

The numbers of pairs $(x, y)$ such that $x \in U_{n}, y \notin U_{n}$ and $a_{x, y} \neq 0$, is smaller than $K 2^{-n}\left|U_{n}\right|$, where $K$ is a positive real number depending only on $G$. Therefore by Jensen's inequality,

$$
\sum_{x \in U_{n}} \sum_{y \notin U_{n}}\left(\left|a_{x, y}\right|^{p}+\left|a_{y, x}\right|^{p}\right) \geq \frac{\left(L_{n}\left|U_{n}\right|\right)^{p}}{\left(K 2^{-n}\left|U_{n}\right|\right)^{p-1}}=\frac{L_{n}^{p}\left|U_{n}\right|}{K^{p-1}\left(2^{-n}\right)^{p-1}}=\frac{\left(2^{n}\right)^{p-1}}{n^{2} K^{p-1}} .
$$

Note that $\left(2^{n}\right)^{p-1} /\left(n^{2} K^{p-1}\right)$ tends to infinity as $n$ tends to infinity, hence $\Phi$ cannot be in $C_{1}^{l_{p}}(G)$.

Now we handle the $c_{0}$-case. Let $\left\{U_{n}\right\}_{n>0}$ and $\delta_{n}$ be as above then $f=\sum_{n} \delta_{n} / n \in$ $C_{0}^{c_{0}}(G)$. Suppose that $f=\partial \Phi$, where $\Phi=\sum a_{x, y}(x, y) \in C_{1}^{c_{0}}(G)$. Then again we have the following estimate:

$$
\frac{1}{n}\left|U_{n}\right| \leq \sum_{x \in U_{n}} \sum_{y \notin U_{n}}\left(\left|a_{x, y}\right|+\left|a_{y, x}\right|\right) \leq \sup _{x, y}\left|a_{x, y}\right| 2 K 2^{-n}\left|U_{n}\right| .
$$

Therefore $\Phi \notin C_{1}^{c_{0}}(G)$.

(Note: A different proof for the $l_{p}$-case was given by Whyte [6].)

\section{Simplicial homologies}

Let $G$ be a nice, directed graph. Now instead of $C_{1}^{l_{r}}(G)$, we consider the space of $p$-summable functions on the edges and denote it by $\widetilde{C}_{1}^{l_{r}}(G)$. The simplicial boundary map $\widetilde{\partial}: \widetilde{C}_{1}^{l_{p}}(G) \rightarrow C_{0}^{l_{p}}(G)$ is defined as usual:

$$
\tilde{\partial} f(x)=\sum_{e=a \rightarrow x} f(e)-\sum_{e=x \rightarrow b} f(e) .
$$

Note that $\widetilde{C}_{1}^{t_{p}}(G) \subset C_{1}^{l_{p}}(G)$ and the boundary map on $\widetilde{C}_{1}^{t_{p}}(G)$ is just the restriction of the boundary map on $C_{1}^{l_{p}}(G)$. The simplicial $l_{p}$-homology, respectively $c_{0}$-homology is defined as

$$
\begin{aligned}
\widetilde{H}_{0}^{l_{p}}(G) & =\widetilde{C}_{0}^{l_{p}}(G) / \widetilde{\partial} \widetilde{C}_{1}^{l_{p}}(G), \\
\widetilde{H}_{0}^{c_{o}}(G) & =\widetilde{C}_{0}^{c_{0}}(G) / \tilde{\partial} \widetilde{C}_{1}^{c_{0}}(G) .
\end{aligned}
$$

A well-known result of Gerl [3] implies that $\widetilde{H}_{0}^{l_{2}}(G)$ is zero if and only if $G$ is non-amenable. We extend this implication. 
THEOREM 2. The following three conditions are equivalent.

(i) $G$ is non-amenable.

(ii) For $p>1, \widetilde{H}_{0}^{l_{p}}(G)=0$.

(iii) $\widetilde{H}_{0}^{c_{0}}(G)=0$.

Proof. If $G$ is amenable, then $\widetilde{H}_{0}^{I_{f}}(G)$ and $\widetilde{H}_{0}^{c_{0}}(G)$ are non-vanishing by Theorem 1 . Now let $G$ be a nice non-amenable graph. Let $f \in C_{0}^{l_{r}}(G)$ and $\Psi \in C_{1}^{l_{p}}(G)$ be such that $\partial \Psi=f$. We construct a $\widetilde{\Psi} \in \widetilde{C}_{1}^{l_{p}}(G)$ such that $\widetilde{\partial} \widetilde{\Psi}=f$.

First of all, for each pair $(a, b) \in \operatorname{supp}(\Psi)$, we choose a path $L_{a, b}$ of minimal length from $a$ to $b$. It is easy to see that there exists a real constant $K>0$, depending only on $\Psi$ such that each edge of $G$ is contained in at most $K$ chosen paths. Now we construct a finitely supported function $\Psi(a, b)$ on the edges for each pair $(a, b), a \neq b$. Let $\Psi_{a, b}(e)=\Psi(a, b)$ if $e$ is in the path $L_{a, b}$ with its own orientation. On the other hand, let $\Psi_{a . b}(e)=-\Psi(a, b)$ if $e$ is in the path $L_{a . b}$ with reverse orientation. Finally, let $\Psi_{a, b}(e)=0$ if the path $L_{a, b}$ does not go through the edge $e$ at all. Then $\Psi_{a, b}$ is supported on at most $M$ edges, where $M$ depends only on $G$, also $\left(\widetilde{\partial} \Psi_{a, b}\right)(b)=\Psi(a, b),\left(\widetilde{\partial} \Psi_{a, b}\right)(a)=-\Psi(a, b)$, otherwise $\widetilde{\partial} \Psi_{a, b}=0$. By our previous observation, $\widetilde{\Psi}=\sum_{(a, b) \in \text { supp } \Psi} \Psi_{a, b}$ is a well-defined element of $\widetilde{C}_{1}^{l_{r}}(G)$ and $\widetilde{\partial} \widetilde{\Psi}=f$. The $c_{0}$-case can be handled the same way.

As an immediate corollary of Theorem 2 we can obtain the simplicial analogue of Gromov's theorem on the vanishing of the $n$th $l_{\infty}$-cohomology of a non-compact $n$-dimensional Riemannian manifold.

Let $K$ be an infinite, connected, oriented $n$-dimensional combinatorial manifold. This means that $K$ is the union of oriented $n$-simplices such that any $(n-1)$-simplex of $K$ is the face of exactly two $n$-simplices with different orientations. Also, the dual graph of $K$ is nice. Recall that the vertices of the dual graph are the $n$-simplices of $K$ and two $n$-simplices are defined adjacent if they have a common $(n-1)$-face. Then one can define the $n$-th simplicial $l_{p}$-cohomology of $K, H_{(p)}^{n}(K)$ as in [5].

Proposition 4.1. Let $K$ be as above. Then $H_{(p)}^{n}(K)=0$ if and only if the dual graph of $K$ is non-amenable.

\section{Translation invariant linear functionals}

Let $G$ be a nice graph. We call a bijection $T: V(G) \rightarrow V(G)$ a translation if there exists a constant $L>0$ such that $d_{G}(x, T(x))<L$ for all $x \in V(G)$. The translations form a group $T(G)$. Note that if $G$ is the Cayley graph of a finitely generated group $\Gamma$, then for any $\gamma \in \Gamma, T_{\gamma}: x \rightarrow x \gamma$ is a translation. Thus we have an injective homomorphism from $\Gamma$ to $T(G)$. For all the Banach spaces we investigated, namely 
$l_{\infty}(G), l_{p}(G)$ and $c_{0}(G)$, the group $T(G)$ can be faithfully represented by the left regular action the following way. If $f \in l_{\infty}(G)$ then $(T f)(x)=f\left(T^{-1}(x)\right)$. Let $B \subset l_{\infty}(G)$ be a $T(G)$-invariant subspace (for example, $l_{p}(G), c_{0}(G)$ ), then we call $\Phi: B \rightarrow \mathbb{R}$ a translation invariant linear functional if it commutes with the $T(G)$-action.

According to Willis $[7,8]$ and Woodward [9], if $\Gamma$ is a finitely generated group, then there exist non-zero $\Gamma$-invariant linear functionals on $l_{p}(G)(p>1)$ and $c_{0}(G)$ if and only if $G$ is amenable. We prove the analogue of their results for arbitrary nice graphs.

THEOREM 3. Let $G$ be a nice amenable graph and let $\alpha: H_{0}^{l_{p}} \rightarrow \mathbb{R}$ be a linear map. Then $\alpha^{*}: f \rightarrow \alpha[f]$ is a translation invariant linear functional on $l_{p}(G)$. The analogous statement holds for $c_{0}(G)$ as well.

PROOF. It is enough to prove that if $T$ is a translation and $f \in l_{p}(G)$, then $T f-f$ is always a 0-boundary. Let $\Psi \in C_{1}^{l_{r}}(G)$ be defined by $\Psi=\sum a_{x, y}(x, y)$, where $a_{x, y}=f(x)$ if $y=T^{-1}(x)$, otherwise $a_{x, y}=0$. Then $T f-f=\partial \Psi$.

Now we prove the complementary result for non-amenable graphs.

THEOREM 4. Let $G$ be a nice, non-amenable graph. Then any translation invariant linear functional on $l_{p}(G)$ and $c_{0}(G)$ is zero.

We will use the following easy-to-prove 'colouring' lemma.

LEMMA 5.1. Let $H$ be a graph such that $k \geq \operatorname{deg}(x)$ for any $x \in V(H)$. Then there exists a function $\pi: E(H) \rightarrow\{1,2, \ldots, 2 k-1\}$ such that if $e$ and $f$ have a joint vertex, then $\pi(e) \neq \pi(f)$.

PROOF (of Theorem 4). Suppose that $\alpha(f) \neq 0$, where $\alpha$ is a translation invariant linear functional on $l_{p}(G)$ and $f \in l_{p}(G)$. By Theorem 1, there exists $\Psi \in C_{1}^{l_{p}}(G)$, $\Psi=\sum a_{x, y}^{\psi}(x, y)$ such that $\partial \Psi=f$. Choose a constant $c>0$ such that $a_{x, y}^{\Psi}=0$ if $d_{G}(x, y)>c$. Now let $H$ be the graph, obtained from $G$ in the following way: $V(H)=V(G)$ and $(x, y) \in E(H)$ if $d_{G}(x, y) \leq c$. Obviously $H$ is nice. We denote the bound on its vertex degrees by $b_{H}$. Let $\pi: E(H) \rightarrow\left\{1,2, \ldots, 2 b_{H}-1\right\}$ as in the previous lemma. Now we can define the translations $T_{i}$ for $1 \leq i \leq 2 b_{H}-1$. Let $T_{i}(x)=y$ if there exists an edge $(x, y) \in H$ such that $\pi(x, y)=i$ and let $T_{i}(x)=x$ otherwise. It is easy to check that all $T_{i}$ 's defined above are in fact translations. Also, if $0<d_{G}(x, y) \leq c$ then there exists exactly one $i, 1 \leq i \leq 2 b_{H}-1$, such that $T_{i}(x)=y$. We define $g_{i} \in l_{p}(G)$ as follows. Let $g_{i}(x)=\Psi(x, y)$ if there exists a 
vertex $y$ such that $(x, y) \in E(H)$ and $\pi((x, y))=i$. Otherwise let $g_{i}(x)=\Psi(x, x)$. Then,

$$
\sum_{i=1}^{2 b_{H}-1}\left(T_{i} g_{i}-g_{i}\right)(x)=f(x)
$$

since $f(x)=\sum_{y}(\Psi(y, x)-\Psi(x, y))$. Then

$$
0 \neq \alpha(f)=\sum_{i=1}^{2 b_{H}-1} \alpha\left(T_{i} g_{i}-g_{i}\right)=\sum_{i=1}^{2 b_{H}-1} 0=0
$$

is a contradiction.

\section{References}

[1] J. Block and S. Weinberger, 'Aperiodic tilings, positive scalar curvature and amenability of spaces', J. Amer. Math. Soc. 5 (1992), 907-918.

[2] W. A. Deuber, M. Simonovits and V. T. Sós, 'A note on paradoxical metric spaces', Studia Sci. Math. Hungar. 30 (1995), 17-23.

[3] P. Gerl, 'Random walks on graphs with strong isoperimetric property', J. Theoret. Probab. 1 (1988), 171-188.

[4] M. Gromov, 'Kaehler hyperbolicity and $L^{2}$-cohomology', J. Differential Geom. 33 (1991), 237263.

[5] M. Gromov, Asymptotic invariants of infinite groups, London Math. Soc., Lecture Note Ser. 182 (1993).

[6] K. Whyte, 'Amenability, bilipschitz equivalence, and the von Neumann conjecture', preprint, University of Chicago, (1997).

[7] G. A. Willis, 'Translation invariant functionals on $L^{P}(G)$, when $G$ is not amenable', $J$. Austral. Math. Soc. (Ser. A) 41 (1986), 237-250.

[8] G. A. Willis, 'Continuity of translation invariant linear functionals on $C_{0}(G)$ for certain locally compact groups $G$ ', Monatsh. Math. 105 (1988), 161-165.

[9] G. S. Woodward, 'Translation-invariant linear forms on $C_{0}(G), C(G), L^{p}(G)$ for non-compact groups', J. Funct. Anal. 16 (1974), 205-220.

\section{Mathematical Institute}

Hungarian Academy of Science

Budapest 1364

POB 127

Hungary 